\title{
Impactor Flux on the Pluto-Charon System
}

\author{
Gonzalo C. de Elía, Romina P. Di Sisto and Adrián Brunini \\ Facultad de Ciencias Astronómicas y Geofísicas - UNLP \\ IALP - CCT La Plata - CONICET \\ Paseo del Bosque S/N (1900), La Plata, Buenos Aires, Argentina \\ email: gdeelia@fcaglp.unlp.edu.ar, romina@fcaglp.unlp.edu.ar \\ abrunini@f caglp.unlp.edu.ar
}

\begin{abstract}
In this work, we study the impactor flux on Pluto and Charon due to the collisional evolution of Plutinos. To do this, we develop a statistical code that includes catastrophic collisions and cratering events, and takes into account the stability and instability zones of the 3:2 mean motion resonance with Neptune. Our results suggest that if 1 Pluto-sized object is in this resonance, the flux of $D=2 \mathrm{~km}$ Plutinos on Pluto is $\sim 4-24$ percent of the flux of $D=2$ $\mathrm{km}$ Kuiper Belt projectiles on Pluto. However, with 5 Pluto-sized objects in the resonance, the contribution of the Plutino population to the impactor flux on Pluto may be comparable to that of the Kuiper Belt. As for Charon, if 1 Pluto-sized object is in the 3:2 resonance, the flux of $D=2 \mathrm{~km}$ Plutinos is $\sim 10-63$ percent of the flux of $D=2 \mathrm{~km}$ impactors coming from the Kuiper Belt. However, with 5 Pluto-sized objects, the Plutino population may be a primary source of the impactor flux on Charon. We conclude that it is necessary to specify the Plutino size distribution and the number of Pluto-sized objects in the 3:2 Neptune resonance in order to determine if the Plutino population is a primary source of impactors on the Pluto-Charon system.
\end{abstract}

Keywords. Kuiper Belt, Solar System: General, Methods: Numerical

\section{Introduction}

The 3:2 mean motion resonance with Neptune, located at $\sim 39.5$ AU, is the most densely populated one in the Kuiper Belt. The residents of this resonant region are usually called Plutinos because of the analogy of their orbits with that of Pluto, which is its most representative member. Aside from Pluto and its largest moon Charon, the Minor Planet Center (MPC) database contains 200 Plutino candidates.

Weissman \& Stern (1994) estimated current impact rates of comets on Pluto and Charon. They showed that cratering is dominated by Kuiper Belt and inner Oort cloud comets. Then, Durda \& Stern (2000) calculated collision rates in the Kuiper Belt and Centaur region. They estimated that the flux of Kuiper Belt projectiles onto Pluto and Charon is $\sim 3-5$ times that of Weissman \& Stern (1994). Later, Zahnle et al. (2003) studied the cratering rates for the moons of the jovian planets and Pluto produced mainly by ecliptic comets, obtaining results consistent with previous estimates.

In this work, we study the impactor flux on Pluto and Charon due to the collisional evolution of Plutinos. Our main goal is to determine if the Plutino population can be considered a primary source of impactors on the Pluto-Charon system.

\section{The Full Model}

In order to simulate the collisional and dynamical evolution of the Plutino population, we use the statistical code developed by de Elía et al. (2008). This algorithm considers 
catastrophic collisions and cratering events, and takes into account the main dynamical characteristic associated to the 3:2 mean motion resonance with Neptune.

\subsection{Initial Populations}

From Kenyon et al. (2008), the cumulative size distribution of the resonant population of the trans-Neptunian region shows a break at a diameter $D$ near $40-80 \mathrm{~km}$. Moreover, for larger resonant objects, the population seems to have a shallow size distribution with a cumulative power-law index of $\sim 3$. On the other hand, Kenyon et al. (2008) suggested that the resonant population has $\sim 0.01-0.05 M_{\oplus}$ in objects with $D \gtrsim 20-40 \mathrm{~km}$. From these estimates, it is possible to infer the existence of 5 Pluto-sized objects in the whole resonant population. If these 5 Pluto-sized objects were all in the 3:2 Neptune resonance we would have an upper limit for the large objects in this resonance.

From this, the general form of the cumulative initial population used in our model to study the collisional and dynamical evolution of the Plutinos can be written as follows

$$
\begin{aligned}
& N(>D)=C_{1}\left(\frac{1 \mathrm{~km}}{D}\right)^{p} \text { for } D \leqslant 60 \mathrm{~km}, \\
& N(>D)=C_{2}\left(\frac{1 \mathrm{~km}}{D}\right)^{3} \text { for } D>60 \mathrm{~km},
\end{aligned}
$$

where $C_{2}$ adopts values of $7.9 \times 10^{9}$ and $3.9 \times 10^{10}$ for 1 and 5 Pluto-sized objects in the 3:2 Neptune resonance, respectively. Given the uncertainty in the parameters of the Plutino size distribution at small sizes, we decide to use in our model three different initial populations, which are defined as follows

- Initial Population 1, with a cumulative power-law index $p$ of 3.0 for $D \leqslant 60 \mathrm{~km}$,

- Initial Population 2, with a cumulative power-law index $p$ of 2.7 for $D \leqslant 60 \mathrm{~km}$,

- Initial Population 3, with a cumulative power-law index $p$ of 2.4 for $D \leqslant 60 \mathrm{~km}$.

\subsection{Collisional Parameters}

Here, we adopt constant values of the intrinsic collision probability $\left\langle P i_{c}\right\rangle$ and the mean impact velocity $\langle V\rangle$ for Plutinos derived by Dell'Oro et al. (2001). Based on a sample of 46 Plutinos, these authors computed values of $\left\langle P i_{c}\right\rangle$ and $\langle V\rangle$ of $4.44 \pm 0.04 \times 10^{-22}$ $\mathrm{km}^{-2} \mathrm{yr}^{-1}$ and $1.44 \pm 0.71 \mathrm{~km} \mathrm{~s}^{-1}$, respectively.

As for the impact strength, we use a combination of the shattering impact specific energy $Q_{S}$ and the inelasticity parameter $f_{k e}$ that yield the impact energy required for dispersal $Q_{D}$ derived by Benz \& Asphaug (1999) for icy bodies at $3 \mathrm{~km} \mathrm{~s}^{-1}$. The $Q_{S}$ law used in our simulations can be represented by an expression of the form

$$
Q_{S}=C_{1} D^{-\lambda_{1}}\left(1+\left(C_{2} D\right)^{\lambda_{2}}\right),
$$

where $C_{1}, C_{2}, \lambda_{1}$, and $\lambda_{2}$ are constant coefficients whose values are 24, 1.2, 0.39 and 1.75 , respectively. Once the $Q_{S}$ law is specified, we adjust the ineslaticity parameter $f_{k e}$ to get the Benz \& Asphaug (1999) $Q_{D}$ law. According to O'Brien \& Greenberg (2005), we express the parameter $f_{k e}$ as

$$
f_{k e}=f_{k e_{0}}\left(\frac{D}{1000 k m}\right)^{\gamma},
$$

where $f_{k e_{0}}$ is the value of $f_{k e}$ at $1000 \mathrm{~km}$ and $\gamma$ is a given exponent. Our simulations indicate that the $Q_{D}$ law from Benz \& Asphaug (1999) for icy bodies at $3 \mathrm{~km} \mathrm{~s}^{-1}$ is obtained with good accuracy from the combination of the selected $Q_{S}$ law and $f_{k e}$, with $f_{k e_{0}}=0.27$ and $\gamma=0.7$. Such values are consistent with those from Davis et al. (1989). 


\subsection{Dynamical Considerations}

To study the orbital space occupied by the Plutino population, we develop a numerical integration of 197 Plutino candidates extracted from the Minor Planet Center database with semimajor axes between 39 and 40 AU. These objects are assumed to be massless particles subject to the gravitational field of the Sun (including the masses of the terrestrial planets) and the perturbations of the four giant planets. The simulation is performed with the simplectic code EVORB from Fernández et al. (2002). The evolution of the test particles is followed for $10^{7}$ years which is a timescale greater than any secular period found in this resonance (Morbidelli 1997). From this, we build maps of the distribution of Plutinos in the orbital element planes $(a, e)$ and $(a, i)$, which allows us to determine the main stability regions of the 3:2 Neptune resonance. Such maps are used to assign a characteristic orbit for every colliding Plutino and to specify the final fates of the different fragments generated in the collisional evolution (see de Elía et al. 2008).

\section{Results}

Figures 1 a) and b) show the number of Plutino impacts onto Pluto over 4.5 Gyr as a function of impactor diameter, when 1 and 5 Pluto-sized objects are present in the 3:2 Neptune resonance, respectively. From the Initial Populations 3, 2 and 1, the total number of $D=2 \mathrm{~km}$ Plutinos impacting Pluto over $4.5 \mathrm{Gyr}$ is $\sim 4.6 \times 10^{2}, 1.1 \times 10^{3}$, and $2.7 \times 10^{3}$, respectively, assuming 1 Pluto-sized object in the resonance. With 5 Plutosized objects, the number of such impacts ranges from $\sim 1.8 \times 10^{3}$ to $9.2 \times 10^{3}$. On the other hand, our simulations indicate that the largest Plutino that impacted Pluto over the age of the Solar System had a diameter of $\sim 26-32 \mathrm{~km}$, if 1 Pluto-sized object is assumed to be present in the resonance. With 5 Pluto-sized objects, the diameter of the largest Plutino that impacted Pluto over 4.5 Gyr is $\sim 44-51 \mathrm{~km}$. Figures 1 a) and b) also show the number of Kuiper Belt object (KBO) impacts onto Pluto over 4.5 Gyr derived by Durda \& Stern (2000). According to this work, the number of $D=2 \mathrm{~km} \mathrm{KBOs}$ striking Pluto over 4.5 Gyr is $\sim 10^{4}$, while the largest KBO expected to have impacted Pluto over the Solar System history had a diameter of $\sim 90 \mathrm{~km}$. Thus, if 1 Pluto-sized object is present in the resonance, the flux of $D=2 \mathrm{~km}$ Plutinos on Pluto is found to be $\sim 4-24$ percent of the flux of $D=2 \mathrm{~km}$ Kuiper Belt projectiles on Pluto. However, if 5 Pluto-sized objects are assumed to be present in the resonance, the contribution of the Plutino population to the impactor flux on Pluto may be comparable to that of the Kuiper Belt.

Figures 2 a) and b) show the number of Plutino impacts onto Charon over 4.5 Gyr as a function of impactor diameter, when 1 and 5 Pluto-sized objects are present in the 3:2
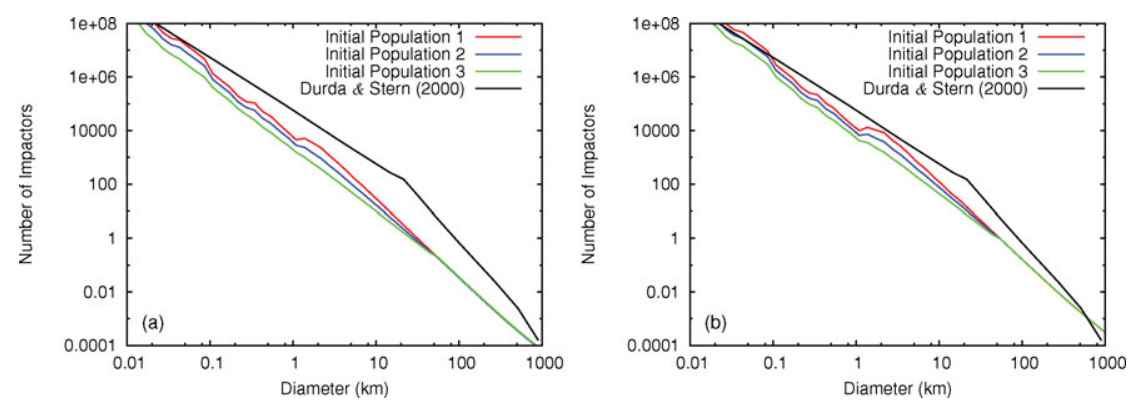

Figure 1. Number of Plutino impacts on Pluto over 4.5 Gyr as a function of impactor diameter, assuming $1 \mathrm{a}$ ) and $5 \mathrm{~b}$ ) Pluto-sized objects in the 3:2 Neptune resonance. 

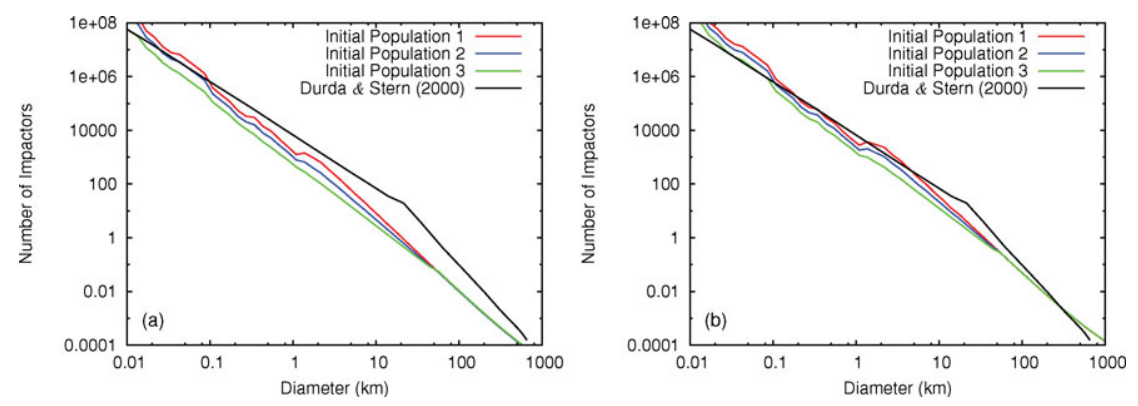

Figure 2. Number of Plutino impacts on Charon over 4.5 Gyr as a function of impactor diameter, assuming $1 \mathrm{a}$ ) and $5 \mathrm{~b}$ ) Pluto-sized objects in the 3:2 Neptune resonance.

Neptune resonance, respectively. With 1 Pluto-sized object, the total number of $D=2$ $\mathrm{km}$ Plutinos impacting Charon over 4.5 Gyr is $\sim 1.3 \times 10^{2}, 3.1 \times 10^{2}$ and $7.6 \times 10^{2}$, from the Initial Populations 3, 2 and 1, respectively. With 5 Pluto-sized objects, the number of such impacts ranges from $\sim 5.1 \times 10^{2}$ to $2.6 \times 10^{3}$. Moreover, our simulations indicate that if 1 Pluto-sized object is in the resonance, the diameter of the largest Plutino that impacted Charon over 4.5 Gyr is $\sim 15-20 \mathrm{~km}$. With 5 Pluto-sized objects, the largest Plutino that impacted Charon over the Solar System history had a diameter of $\sim 30-35$ $\mathrm{km}$. Figures $2 \mathrm{a}$ ) and b) also show the number of Kuiper Belt object (KBO) impacts onto Charon over 4.5 Gyr from Durda \& Stern (2000). From this, the number of $D=2 \mathrm{~km}$ KBOs striking Charon over $4.5 \mathrm{Gyr}$ is $\sim 1.2 \times 10^{3}$, while the largest KBO expected to have impacted Charon over the Solar System history had a diameter of $\sim 50 \mathrm{~km}$. Thus, if 1 Pluto-sized object is present in the resonance, the flux of $D=2 \mathrm{~km}$ Plutinos on Charon is found to be $\sim 10-63$ percent of the flux of $D=2 \mathrm{~km}$ impactors on Charon coming from the Kuiper Belt. However, if 5 Pluto-sized objects are assumed in the resonance, the Plutino population may be a primary source of the impactor flux on Charon.

Our results depend strongly on the initial size distribution and primarily on the number of Pluto-sized objects present in the 3:2 Neptune resonance. Thus, we conclude that it is very necessary to know the Plutino size distribution and the number of Pluto-sized objects in this resonant region in order to determine if the Plutino population can be considered a primary source of impactors on the Pluto-Charon system.

\section{References}

Benz, W. \& Asphaug, E. 1999, Icarus, 142, 5

Davis, D. R., Weidenschilling, S. J., Farinella, P., Paolicchi, P., \& Binzel, R. P. 1989, in: R. P. Binzel, T. Gehrels \& M. S. Matthews (eds.), Asteroids II, (Tucson: University of Arizona Press), p. 805

de Elía, G. C., Brunini, A., \& Di Sisto, R. P. 2008, A\& $A$, 490, 835

Dell'Oro, A., Marzari, F., Paolicchi, P., \& Vanzani, V. 2001, A\&AA, 366, 1053

Durda, D. D. \& Stern, S. A. 2000, Icarus, 145, 220

Fernández, J. A., Gallardo, T., \& Brunini, A. 2002, Icarus, 159, 358

Kenyon, S. J., Bromley, B. C., O'Brien, D. P., \& Davis, D. R. 2008, in: M. A. Barucci, H. Boehnhardt, D. P. Cruikshank, \& A. Morbidelli (eds.), The Solar System Beyond Neptune, (Tucson: University of Arizona Press), p. 293

Morbidelli, A. 1997, Icarus, 127, 1

O'Brien, D. P. \& Greenberg, R. 2005, Icarus, 178, 179

Zahnle, K., Schenk, P., Levison, H., \& Dones, L. 2003, Icarus, 163, 263 